\title{
Identification and Quantification of the Left-to-Right Shunt in Adults with Atrial Septal Defect by Two-Dimensional Negative Contrast Echocardiography
}

\author{
Hidezo Mori, M.D., Satoshi OGawa, M.D., \\ Masako Toyama, M.D., Masao Sano, M.D., Takeshi Tsugu, M.D., \\ Shunnosuke Handa, M.D., and Yoshiro Nakamura, M.D.
}

\section{SUMMARY}

Negative contrast echocardiograms of two-dimensional long axis view of the right ventricle and the atrium were compared with the results of cardiac catheterization and surgical findings in 33 adults with atrial septal defects. Contrast echocardiographic evidence of atrial communication was noted in 30 cases $(91 \%)$, and, in 28 cases, the negative contrast during one cardiac cycle could be measured. This could be applied even in cases with a small left-to-right shunt or with Eisenmenger's syndrome. No obvious difference in the behavior of negative contrast was documented among the cases with distinct types of septal defects.

The maximum negative contrast area and the duration of appearance in one cardiac cycle were related to the pulmonary-tosystemic flow ratio. However, a significant overlap and considerable effects of transducer angulation on measurements were documented. Thus, negative contrast echocardiography may be widely used for the noninvasive identification of a left-to-right shunt in atrial septal defects. However, the method is not appropriate for a precise quantification of three-dimensional shunt flow.

\section{Additional Indexing Words:}

Contrast echocardiography Atrial septal defect Left-to-right shunt Pulmonary-to-systemic flow ratio Oximetry Twodimensional echocardiography

CONTRAST echocardiography was originally introduced by Gramiak et $1 a^{11}$ for the identification of various intracardiac structures. It has become an important procedure to evaluate congenital heart disease with an intracardiac shunt or valvular regurgitation. ${ }^{2)-4)}$ Recently, the value of so-cal-

From the Cardiopulmonary Division, Department of Medicine, Keio University, Tokyo, Japan.

Address for reprint: Hidezo Mori, M.D., Cardiopulmonary Division, Department of Medicine, Keio University, 35 Shinanomachi, Shinjuku-ku, Tokyo 160, Japan.

Received for publication April 5, 1983. 
led negative contrast images in the right atrium was emphasized for direct visualization of a left-to-right shunt in cases with atrial septal defects. ${ }^{5)-7)}$ In the present study, the utility and limitations of negative contrast echocardiography for the identification of atrial septal defects and the estimation of the left-to-right shunt degree were examined.

\section{Materials and Methods}

Two-dimensional contrast echocardiograms from 33 adults (18-58 yrs old, $41.0 \pm 9.9$ in mean) with atrial septal defects (29 secundum type and 4 primum type) were analyzed prior to cardiac catheterization and compared with the results of oximetry and surgical findings. The subjects consisted of 13 males and 20 females. Thirty-one of 33 cases had a normal sinus rhythm and the remaining 2 had atrial fibrillation with relatively fixed $R-R$ intervals under digitalis.

Contrast echocardiography:

Two-dimensional echocardiography was performed using a phased array imaging system (Toshiba SSH 11-A) and video cassettes (SONY KCA 60A). A parasternal long axis view of the right ventricle and the right atrium was obtained from the patients in the $30^{\circ}$ left lateral position. In the setting of the echocardiographic plane, care was taken to visualize the defective portion of the interatrial septum and to include the anterior leaflet of the tricuspid valve within the plane.

Twenty $\mathrm{ml}$ of pre-cooled and pre-shaken saline was injected rapidly through a 19 gauge elastic needle placed in the antecubital vein to obtain contrast images. The patient was asked to hold his breath at the end-expirium throughout the recording. Negative contrast images in the right atrium, which were completely encircled by the contrast materials and continuous to the defective portion of the interatrial septum, were measured during one cardiac cycle on the face of a TV screen by using a single frame presentation.

To delineate the shunt dynamics, each cardiac cycle was divided into six phases in relation to the simultaneously recorded electrocardiogram and the motion of the tricuspid valve; that is, early, mid, and late systoles and diastoles. The largest and well demarcated negative contrast area in each phase was selected for the measurements. The numbers of phases with a negative contrast appearance and maximum negative contrast area throughout one cardiac cycle (maximum negative contrast) were used as indices of the shunt degree.

In 15 cases, several contrast injections (at least 3) were given while observations of multiple long axis views of the right ventricle and right atrium 
were carried out, with slight angulation of the beam plane across the septal defect. The series in which the negative contrast image was maximally visualized was used for the statistical analysis. In 18 cases comprising the early series of this protocol, trials under multiple transducer angulation were lacking. The heart rates during the procedures ranged from 58 to 120 per minute (83.4 \pm 12.9 , mean \pm standard deviation).

Cardiac catheterization:

Invasive studies were performed no later than 4 days after the echocardiographic examination. Pressure measurement, oximetry, cardiac output measurement by Fick's method, left ventriculography and pulmonary arteriography were included in the studies. The pulmonary-to-systemic flow ratios were calculated in all patients, using oxygen saturation of mixed venous blood as determined by the following formula. ${ }^{81}$

Mixed vein $\mathrm{O}_{2}$ saturation $=$

$$
\frac{\begin{array}{c}
\text { Inferior vena cava } \\
\mathrm{O}_{2} \text { saturation }
\end{array} \times 2+{ }_{\text {Superior vena cava }}^{\mathrm{O}_{2} \text { saturation }} \times 1}{3}
$$

\section{RESULTS}

\section{Identification of left-to-right shunt:}

Negative contrast areas during one cardiac cycle could be measured in 28 of the 33 cases. A representative example from a case with a secundum defect and a dominant left-to-right shunt is shown in Fig. 1. A small negative contrast area of $1.5 \mathrm{~cm}^{2}$ appeared in the early systole (left), then disappeared immediately; the contrast materials filled the entire right atrium in the mid-systole (mid). A larger negative contrast area reappeared from the late systole to the early diastole $\left(3.5 \mathrm{~cm}^{2}\right)$. After a gradual size decrease during the mid-diastole, a second surge was noted following atrial contraction and the maximum value of $3.9 \mathrm{~cm}^{2}$ was noted.

As indicated in Table I, the patients were classified into 4 sub-groups based on the results of oximetry. Association of anomalous pulmonary venous drainage was not recognized on the pulmonary arteriograms in any of the patients. Negative contrast could be measured by planimetry in 6 of the 7 cases with a small left-to-right shunt, and even in 1 case in which significant step up of $\mathrm{O}_{2}$ saturation was not documented, but pulmonary arteriography revealed evidence of an intracardiac left-to-right shunt. A negative contrast with a short duration of appearance was even documented in all 4 cases of Eisenmenger's syndrome (the upper panel of Fig. 2). However, in these cases, an encroachment of the contrast materials into the left atrium was 


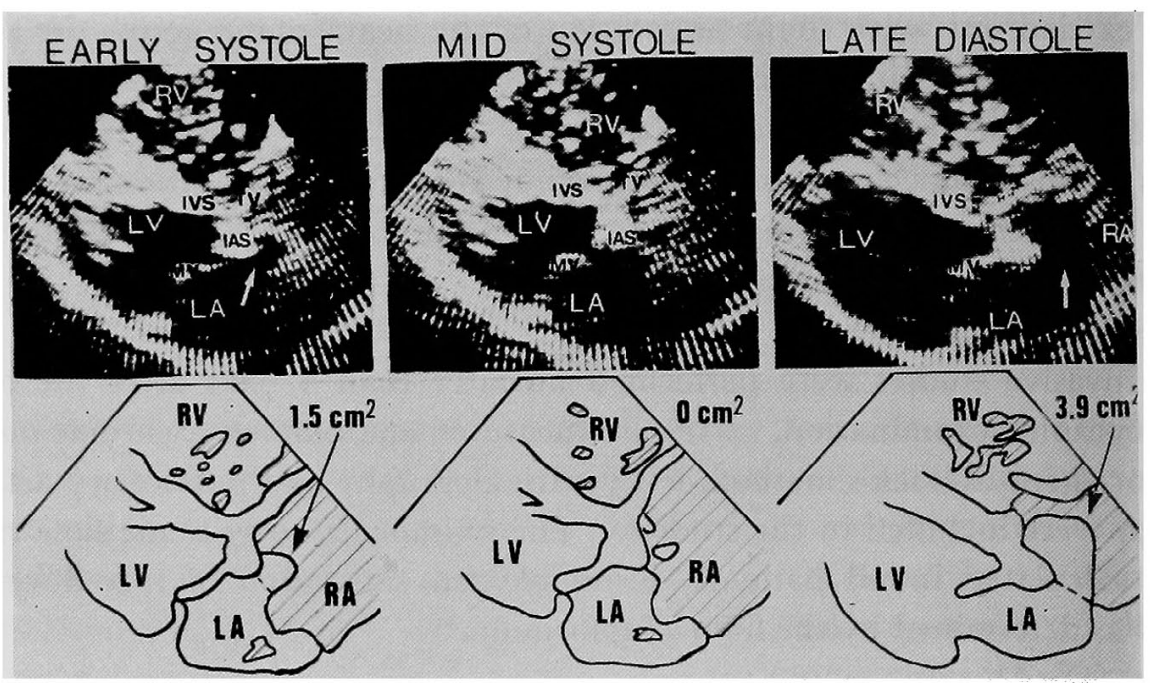

Fig. 1. Representative sequence of negative contrast during one cardiac cycle. Negative contrast echocardiograms in the early systole (left panel), mid systole (middle panel), and late diastole (right panel) in a case with a dominant left-to-right shunt (pulmonary-to-systemic flow ratio of 2.6 and left-to-right shunt flow of $11.8 \mathrm{~L} / \mathrm{min}$ ) are indicated. Contrast materials in the right atrium and ventricle is indicated by oblique lines in schematic representations.

Abbreviations: IAS = interatrial septum; IVS = interventricular septum; $\mathrm{LA}=$ left atrium; $\mathrm{LV}=$ left ventricle; $\mathrm{RA}=$ right atrium; $\mathrm{RV}=$ right ventricle; $\mathrm{TV}=$ tricuspid valve; $\mathrm{MV}=$ mitral valve.

Table I. Summary of the Results of Cardiac Catheterization

\begin{tabular}{l|c|c|c|c}
\hline & $\begin{array}{c}\text { Number } \\
\text { of cases }\end{array}$ & $\begin{array}{c}\text { L-R flow } \\
(\mathrm{L} / \mathrm{min})\end{array}$ & $\begin{array}{c}\text { PA mean Pr. } \\
(\mathrm{mmHg})\end{array}$ & $\begin{array}{c}\text { PAR } \\
\left(\text { dynes sec } \mathrm{cm}^{-5}\right)\end{array}$ \\
\hline $\begin{array}{l}\text { Large L-R } \\
(\mathrm{Qp} / \mathrm{Qs}>2.5)\end{array}$ & 13 & $14.65 \pm 8.07$ & $22.2 \pm 6.7$ & $84.8 \pm 48.7$ \\
$\begin{array}{l}\text { Moderate L-R } \\
(2.0 \leq \mathrm{Q} \text { p/Qs } \leq 2.5)\end{array}$ & 8 & $6.66 \pm 0.79$ & $18.8 \pm 8.9$ & $97.3 \pm 69.1$ \\
$\begin{array}{l}\text { Small L-R } \\
(\mathrm{Qp} / \mathrm{Q}<2.0)\end{array}$ & 7 & $3.58 \pm 0.97$ & $17.8 \pm 9.7$ & $108.7 \pm 61.0$ \\
$\begin{array}{l}\text { Eisenmenger's } \\
\text { syndrome }\end{array}$ & 4 & $\begin{array}{c}1.5,1.6 \\
(\mathrm{n}=2)\end{array}$ & $72.5 \pm 25.9$ & $\begin{array}{c}1287,1293 \\
(\mathrm{n}=2)\end{array}$
\end{tabular}

Abbreviations: L-R=left-to-right shunt; PA mean Pr.=mean pressure of pulmonary artery; $\mathrm{PAR}=$ pulmonary arterial resistance; $\mathrm{Qp} / \mathrm{Qs}=$ pulmonary-to-systemic flow ratio.

a more prominent observation (the lower panel of Fig. 2). In the cases with a primum defect, the sequence of negative contrast appearance was similar with those in secundum defect cases. However, an extension of the negative contrast region into the right ventricle beyond the tricuspid valve was often noted.

In general, the maximum negative contrast was noted either in the late 


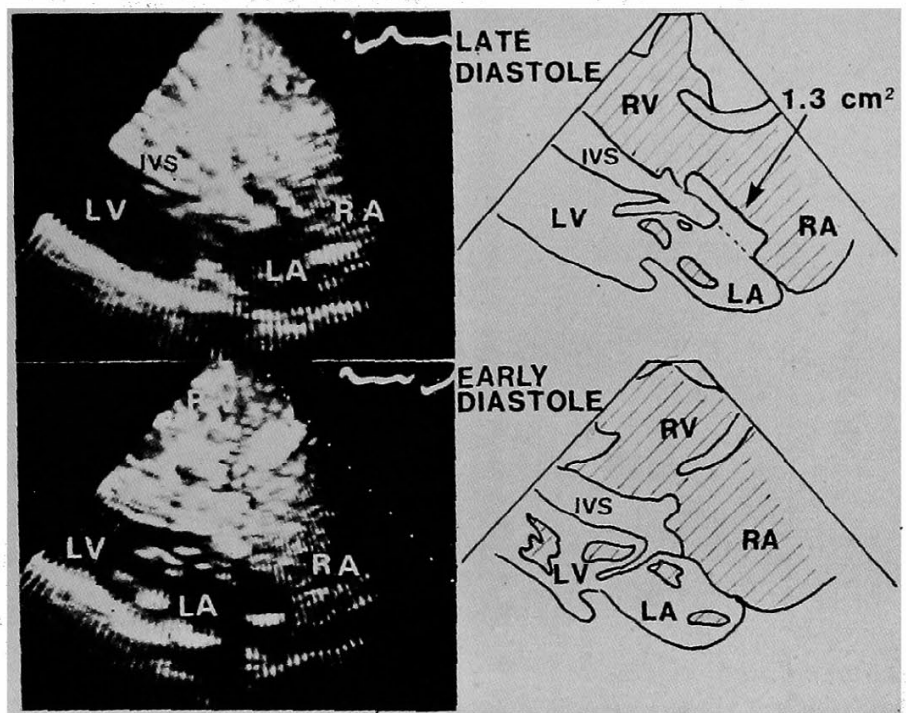

Fig. 2. Contrast echocardiograms in a patient with Eisenmenger's syndrome. In this case, although a small negative contrast image is noted in the late diastole (upper panel), contrast encroachment into the left atrium was a more prominent observation throughout the cardiac cycle (lower panel).

Abbreviations: IVS=interventricular septum; LA =left atrium; $L V=$ left ventricle; $\mathrm{RA}=$ right atrium; $\mathrm{RV}=$ right ventricle.

systole (11 cases), in the early diastole (13 cases), or in the late systole (3 cases). In 1 case with a maximum negative contrast in the mid-systole, massive mitral regurgitation by which the left atrium was entirely opacified was documented on the left ventriculogram.

Semiquantitative assessment of shunt degree:

As shown in Fig. 3, a negative contrast region appeared in five or more different phases in 7 of 9 cases with a large left-to-right shunt, and in four phases or less in 5 of 6 cases with a small left-to-right shunt and in all 4 cases of Eisenmenger's syndrome. The mean area of the maximum negative contrast region in the cases with a small left-to-right shunt was $1.30 \pm 0.50 \mathrm{~cm}^{2}$. This increased significantly to $2.20 \pm 0.77 \mathrm{~cm}^{2}(\mathrm{p}<0.05)$ in the cases with a moderate left-to-right shunt, and a further increase was noted in 9 cases with a large left-to-right shunt $\left(4.57 \pm 1.37 \mathrm{~cm}^{2}, \mathrm{p}<0.005\right)$. The groups of cases with a small left-to-right shunt and with Eisenmenger's syndrome $0.93 \pm$ $0.36 \mathrm{~cm}^{2}$ ) could be easily differentiated by the presence of a massive contrast encroachment from the right atrium into the left atrium in the latter.

Limitations of negative contrast echocardiography:

Considerable variations in negative contrast area were often noted when the transducer angulation was changed slightly. In the particular case shown 


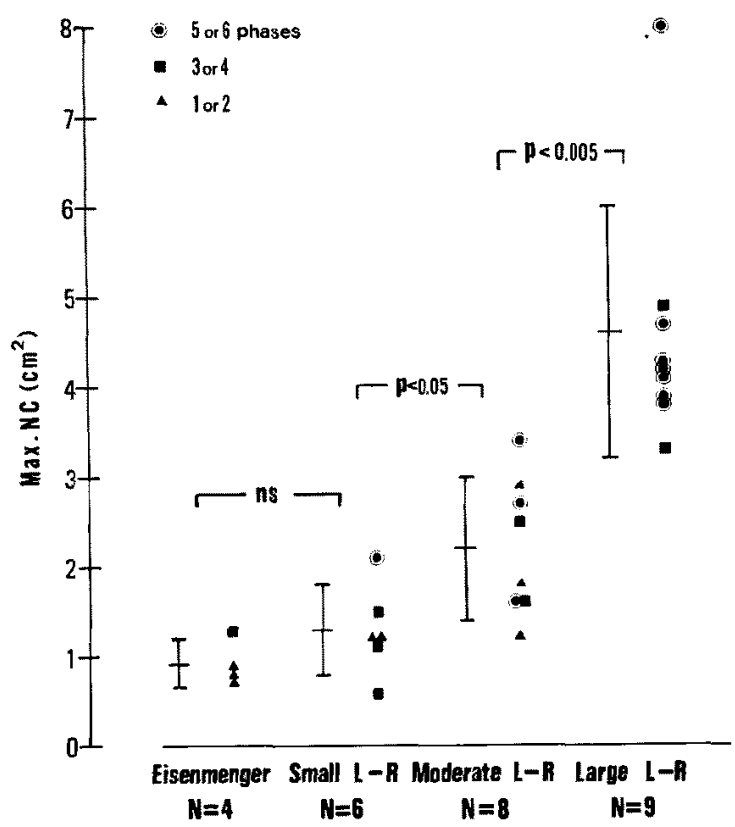

Fig. 3. Comparison of the maximum negative contrast area and numbers of phases of appearance among patients with various degrees of left-toright shunt or bidirectional shunt. The negative contrast area was noted in five phases or more in most of the cases with a large lcft-to-right shunt (double circles). In 3 of the 4 cases with Eisenmenger's syndrome negative contrast appearance is noted in one or two phases (triangles). The vertical axis indicates the maximum negative contrast area.

Abbreviations: L-R=left-to-right shunt; $\mathrm{Max} . \mathrm{NC}=\operatorname{maximum}$ negative contrast area; $\mathrm{ns}=$ not significant.

in Fig. 4, a small negative contrast region $\left(1.5 \mathrm{~cm}^{2}\right)$ was noted only in the late systole on a recording from the usual parasternal position; it was documented in five phases, with a maximum appearance of $4.2 \mathrm{~cm}^{2}$ in the late systole, in the plane viewed from a lower intercostal space. In 3 secundum defect cases (pulmonary-to-systemic flow ratio of $6.3,2.7$, and 1.8 , respectively) of the 18 cases without trials with multiple transducer angulations, neither a negative contrast region in the right atrium nor contrast encroachment into the left atrium was noted.

The characteristics of the surgical findings of septal defects in these 3 cases were compared with 18 other cascs in whom contrast echocardiograms could identify the shunt. The locations of the defects in the interatrial septum varied considerably, even in patients with a secundum defect. Contrast echocardiographic evidence of an atrial communication could not be identified in 2 out of 7 cases with a secundum defect at the upper or lower margins of the interatrial septum and in 1 out of 10 cases with a defect in the 

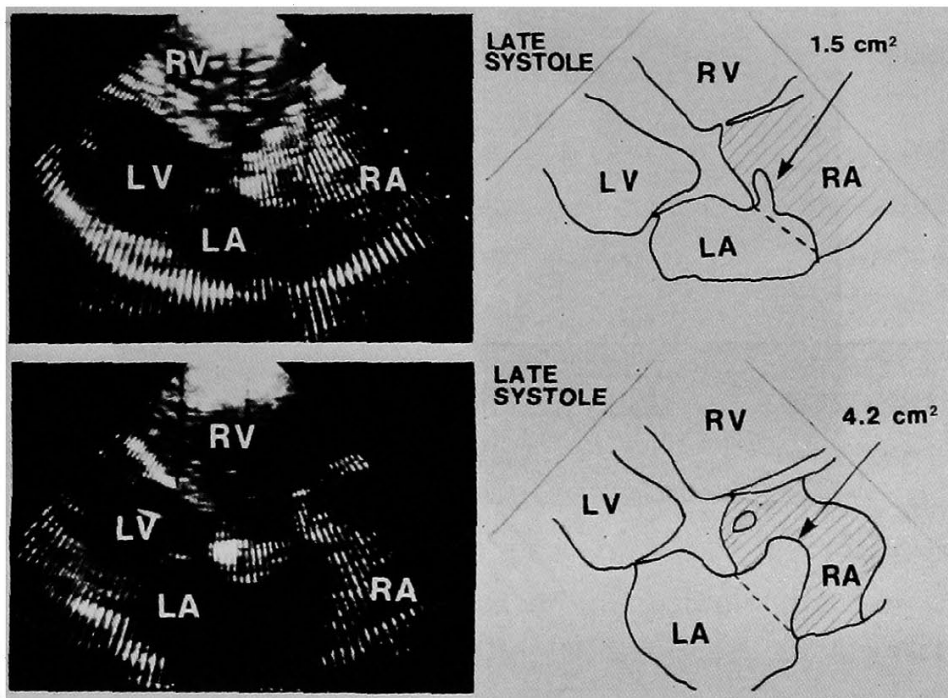

Fig. 4. Variation in behavior of negative contrast under different settings of the echocardiographic plane. In this case with a pulmonary-to-systemic flow ratio of 4.2, negative contrast is noted only in the late systole and the maximum area was $1.5 \mathrm{~cm}^{2}$ on the plane viewed from the usual parasternal approach (upper panel). However, it is identified in five phases during the cardiac cycle with a maximum appearance of $4.2 \mathrm{~cm}^{2}$ on the plane viewed from the lower intercostal space (lower panel).

Abbreviations: $L A=$ left atrium; $L V=$ left ventricle; $R A=$ right atrium; $\mathrm{RV}=$ right ventricle.

central portion of the interatrial septum surrounding the foramen ovale. In the cases with a subtotal defect of the secundum interatrial septum (2 cases) or a primum defect (2 cases), a negative contrast region was successfully visualized. The defect size of the 3 false negative cases $(5 \mathrm{~cm}, 3 \mathrm{~cm}$, and $3 \mathrm{~cm}$ in longitudinal diameter) was within the range of the other 18 cases $(2-7 \mathrm{~cm}$, mean $4.1 \pm 1.4 \mathrm{~cm})$.

In the other 2 cases with a left-to-right shunt, negative contrast areas could not be quantified. However, there was evidence of a communication at the atrial level. In 1 case with a massive left-to-right shunt, shown in Fig. 5 , negative contrast images could be noted in all of the six phases. However, since the negative contrast occupied most of the right atrium, differentiation of negative contrast images due to left-to-right shunt blood from that of venous blood returning from the inferior vena cava or coronary sinus was impossible. In the primum defect case with massive tricuspid regurgitation, indicated by an increased $\mathrm{V}$ wave of $35 \mathrm{mmHg}$, negative contrast could not be demarcated, because contrast materials were scattered within the right atrium by the regurgitant jet. 


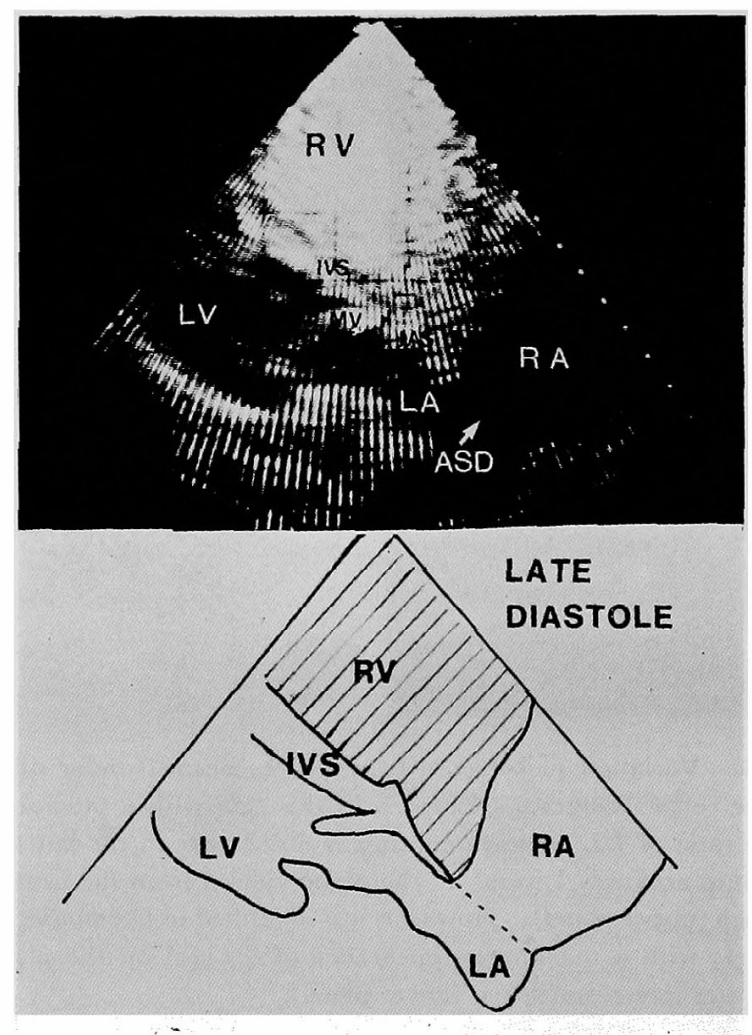

Fig. 5. Negative contrast in a case with a massive left-to-right shunt (pulmonary-to-systemic flow ratio of 6.7 and left-to-right shunt flow of $35 \mathrm{~L} /$ min). Since most of the RA, with the exception of the part adjacent to the intact interatrial septum, is contrast free, negative contrast images resulting from left-to-right shunt flow and from contrast free venous blood cannot be differentiated.

Abbreviations: $A S D=$ defective portion of the secundum interatrial septum; IAS=interatrial septum; IVS=interventricular septum; LA=left atrium; $\mathrm{LV}=$ left ventricle; $\mathrm{MV}=$ mitral valve; $\mathrm{RA}=$ right atrium; $\mathrm{RV}=$ right ventricle.

\section{Discussion}

The usefulness and limitations of negative contrast echocardiography in atrial septal defects with different anatomical and/or hemodynamic characteristics have not yet been fully examined. In the present study, it was demonstrated that the identification of a left-to-right shunt by negative contrast images is feasible in patients with a small left-to-right shunt, including a patient in whom a left-to-right shunt could not be detected by oximetry. Furthermore, the negative contrast images were seen even in patients with a bidirectional or dominant right-to-left shunt. No obvious differences in the be- 
havior of the negative contrast regions were documented among the patients with primum defects or secundum defects with different anatomical characteristics.

The sequence of the shunt in one cardiac cycle can be estimated by using a frame by frame analysis of negative contrast images. It is known that a small pressure gradient between the left and right atria determines the instantaneous direction and the magnitude of the shunt at atrial level.91,10) The maximum left-to-right shunt occurs following the peak of an atrial $\mathrm{V}$ wave and the second peak is noted during atrial contraction..$^{91,10)}$ In the present study, maximum negative contrast was generally identified around the time of tricuspid valve opening, i.e., in the late systole or the early diastole, and the second peak was often noted in the late diastole. Furthermore, an unusual peak of the negative contrast area in mid-systole was noted in a case with massive mitral regurgitation. Thus, the sequence of negative contrast images appeared to reflect the instantaneous pressure difference between both atria.

Furthermore, the semiquantitative relationship of the size of the negative contrast area and its duration with pulmonary-to-systemic flow ratio was documented. However, a significant overlap was also noted. One possible explanation for this overlap is that contrast echocardiography was not performed simultaneously with oximetry in the present study. However, inherent problems of two-dimensional contrast echocardiography may also have a considerable influence. Negative contrast region was not documented in the 3 cases in whom a dominant left-to-right shunt was verified by oxymetry and a secundum defect was identified surgically. Inappropriate settings of the echocardiographic plane may be one reason for the lack of negative contrast documentation, as multiple trials with different transducer angulations were lacking in these cases. In addition, the fact that the negative contrast size varied considerably with the transducer angulation (Fig. 4) further supports this speculation. This emphasizes the importance of multi-angular trials for the identification of the shunt, since negative contrast images are only a two-dimensional visualization of three-dimensional shunt flow.

In order to improve the diagnostic accuracy, certain precautions were also taken in this study. Contrast-containing blood returning from the left atrium sometimes made an accurate demarcation of negative contrast images difficult. Therefore, it was important to select the earliest possible cardiac cycles for evaluation; i.e., the first or second cycle.

The negative contrast created by contrast-free venous blood should also be discussed. In the present study, negative contrast assessment was confined to areas demarcated completely by the contrast materials and continuous with 
the defective portion of the interatrial septum. Therefore, the effects of the blood from the inferior vena cava were minimized. By contrast, the effects of coronary sinus flow cannot be avoided in cases with the shunt pathway in the posterior and lower portions, since the ostium of the coronary sinus lies adjacent to the plane of the interatrial septum. However, the amount of blood flow from the coronary sinus is much smaller than that of shunt flow in an atrial septal defect of clinical importance. In this sense, the effect of the coronary sinus flow may be less important.

\section{REFERENCES}

1. Gramiak R, Shah PM, Kramer DH: Ultrasound cardiography. Contrast studies in anatomy and function. Radiology $92: 939,1969$

2. Seward JB, Tajik AJ, Hagler DJ, Ritter DG: Peripheral venous contrast echocardiography. Am J Cardiol 39: 202, 1977

3. Valdes-Curz LM, Pieroni DR, Roland MA Jr, Varghese PJ : Echocardiographic detection of intracardiac right-to-left shunts following peripheral vein injections. Circulation 54: 558, 1976

4. Lieppe W, Behr VS, Scallion R, Kisslo JA: Detection of tricuspid regurgitation with twodimensional echocardiography and peripheral vein injections. Girculation 57: 128, 1978

5. Weyman AE, Wann LS, Caldwell RL, Hurwitz RA, Dillon JC, Feigenbaum H: Negative contrast echocardiography. A new method for detecting left-to-right shunts. Circulation 59: 498,1979

6. Fraker TD Jr, Harris PJ, Behar VS, Kisslo JA: Detection and exclusion of interatrial shunts by two-dimensional echocardiography and peripheral vein injection. Circulation 59: 379, 1979

7. Dillon JC, Weyman AE, Feigenbaum H, Eggleton RC, Johnston K: Cross-sectional echocardiographic examination of the interatrial septum. Circulation 55: 115, 1977

8. Rowe GG, Castillo CA, Maxwell GM, Clifford JE, Crumpton CW: Atrial septal defect and mechanism of shunt. Am Heart J 61 : 369, 1961

9. Levin AR, Spach MS, Boineau JP, Canent RV Jr, Capp MP, Jewett PH: Atrial pressureflow dynamics in atrial septal defects (secundum type). Circulation 37: 476, 1968

10. Alexander JA, Rembert JC, Sealy WC, Greenfield JG: Shunt dynamics in experimental atrial septal defects. J Appl Physiol 39: 281, 1975 